\title{
ANALYTICITY OF SETS ASSOCIATED TO LELONG NUMBERS AND THE EXTENSION OF MEROMORPHIC MAPS ${ }^{1}$
}

\author{
BY YUM-TONG SIU ${ }^{2}$ \\ Communicated by S. S. Chern, May 1, 1973
}

We announce the following two theorems and sketch the proofs of some special cases which are indicative of the ideas of the complete proofs.

THEOREM I. If $u$ is a closed positive $(k, k)$ current on an open subset of $C^{n}$, then for $c \geqq 0$ the set of points where the Lelong number of $u$ is $\geqq c$ is an analytic set.

THEOREM II. Let $X$ be a normal complex space, $A$ be an analytic set of codimension $\geqq 1$ in $X$, and $G$ be an open subset of $X$ which intersects every branch of $A$ of codimension 1 . If $M$ is a compact Kähler manifold, then every meromorphic map $f$ (in the sense of Remmert) from $(X-A) \cup G$ to $M$ can be extended to a meromorphic map from $X$ to $M$.

Theorem I has been an outstanding conjecture. This author first heard the conjecture mentioned by Lelong in the 1972 Several Complex Variables Conference in Poitiers, France, and later learned that it was also mentioned in [6] and by Harvey in the 1971 Berkeley Summer Conference on Partial Differential Equations. Some partial results in this direction have been obtained. Thie [12] proved that, when $u$ is defined by integration over an analytic subset of pure codimension $k$, the Lelong number agrees with the multiplicity of the analytic set and hence the conjecture is true in this case. King [7] proved that, if the Lelong number of $u$ is an integer at almost every point of its support with respect to the Hausdorff $(2 n-2 k)$ measure, then $u$ is a linear combination of currents defined by integration over analytic subsets of pure codimension $k$, and, as a consequence, the conjecture is true in this case. Harvey and King [6] improved the result of [7] and showed that if, for every compact subset $K$ of Supp $u$, there exists $c>0$ such that the Lelong number of $u$ at $x$ is $\geqq c$ for almost all $x \in K$ with respect to the Hausdorff $(2 n-2 k)$-measure, then $u$ is a linear combination of currents defined by integration over analytic subsets of pure codimension $k$, and, consequently, the conjecture is true in this case. Recently Skoda [11] proved that, if the domain $D$ where $u$ is defined is Stein, then for every $c \geqq 0$ there exists an analytic subset $X_{c}$ of $D$ such that

AMS (MOS) subject classifications (1970). Primary 32C30, 32D15, 53C65; Secondary 32H99, 53C55.

${ }^{1}$ Research partially supported by a NSF grant.

2 Sloan Fellow. 
$E_{c} \subset X_{c} \subset E_{k c / n}$, where $E_{c}$ is the set of points where the Lelong number of $u$ is $\geqq c$.

The special case of Theorem II where $X$ is the open unit ball in $C^{n}$ with $n \geqq 3$ and $A=\{0\}$ was proved by Griffiths [5]. Shiffman [9] relaxed the condition $n \geqq 3$ in Griffiths' result to $n \geqq 2$ (and proved some slightly more general cases where $A$ is compact). The case of Theorem II where the codimension of $A$ is $\geqq 2$ was conjectured by Griffiths [5].

1. We now give a sketch of the proof of Theorem $\mathrm{I}$ when $k=1$ and $n \geqq 2$. We denote the Lelong number of $u$ at $x$ by $n(u, x)$ and let $E_{c}=\{x \mid n(u, x) \geqq c\}$.

(1.1) Lemma. Let $\varphi$ be a plurisubharmonic function on an open subset $D$ of $\boldsymbol{C}^{n}$ and $V$ be a submanifold of codimension 1 in $D$ whose ideal sheaf is generated by a single holomorphic function $f$ on D. Let $u=(\sqrt{-1} / \pi) \partial \bar{\partial} \varphi$. If $V \subset E_{c}$, then $\varphi-c \log |f|$ is plurisubharmonic on $D$.

Proof. By a covering theorem of Vitali type $[4, \mathrm{p} .152$, Theorem 2.8.18], we conclude that the Laplacian of $\varphi-c \log |f|$ is a positive measure and hence $\varphi-c \log |f|$ is subharmonic. Being plurisubharmonic on $D-V$ and locally uniformly bounded from above (due to its subharmonicity), $\varphi-c \log |f|$ is plurisubharmonic on $D$. Q.E.D.

(1.2) Lemma. Let $\varphi, D, V, f, u$ be the same as in (1.1). If $D$ is Stein and $V$ is connected, then for $c \geqq 0$ either $V \subset E_{c}$ or $E_{c} \cap V$ is contained in a proper analytic subset of $V$.

Proof. Let $c_{0}=\inf \{n(u, x) \mid x \in V\}$. By replacing $\varphi$ by $\varphi-c_{0} \log |f|$, we can assume without loss of generality that $c>c_{0}=0$. By Skoda's result [11] or Bombieri's results [2], [3], there exists an analytic subset $X_{c}$ of $D$ such that $E_{c} \subset X_{c} \subset E_{c^{\prime}}$ for some $0<c^{\prime}<c$. Since $n(u, x)<c^{\prime}$ for some $x \in V, X_{c} \cap V$ is a proper analytic subset of $V$. Q.E.D.

Let $u$ be a closed positive $(1,1)$ current on the open unit ball $B$ in $C^{n}$. Then there exists a plurisubharmonic function $\varphi$ on $B$ such that $u=(\sqrt{-1} / \pi) \partial \bar{\partial} \varphi$. For every complex $q$-plane $L$ in $C^{n}$ passing through 0 , define $u \mid L=(\sqrt{-1} / \pi) \partial \bar{\partial}(\varphi \mid L)$ on $L \cap B$ when it is meaningful. $u \mid L$ is defined for almost all complex $q$-plane $L$. By blowing up the point 0 and using (1.1) and (1.2) (and the fact that a Lelong number which is 0 for one local coordinates system is 0 for all local coordinates systems), we obtain the following:

(1.3) Lemma. $n(u, 0)=n(u \mid L, 0)$ for almost all complex lines $L$ through 0.

As a consequence of (1.3) we have: 
(1.4) LEMMA. If the germ of $u$ at 0 is invariant under the action of $U(n-q)$ on $z_{q+1}, \ldots, z_{n}$, then $n(u, 0)=n(u \mid L, 0)$, where $L$ is the complex $(q+1)$-plane defined by $z_{q+2}=\cdots=z_{n}=0$.

From (1.3) and by considering linear coordinates systems first, we obtain:

(1.5) Lemma. $n(u, 0)$ is independent of the local coordinates system at 0.

(1.6) Lemma. Let $D$ be a Stein domain in $C^{q}, B$ be the open unit ball in $C^{n-q}$, and $u$ be a closed positive $(1,1)$ current on $D \times B$. If $c \geqq 0$, then either $D \times\{0\} \subset E_{c}$ or $(D \times\{0\}) \cap E_{c}$ is contained in a proper analytic subset of $D \times\{0\}$.

Proof. The case $q=n-1$ is (1.2). For $q<n-1$, by averaging $u$ over the action of $U(n-q)$ on $z_{q+1}, \ldots, z_{n}$, we can assume without loss of generality that $u$ is invariant under the action of $U(n-q)$ on $z_{q+1}, \ldots, z_{n}$. The result now follows from (1.4) and applying (1.2) to

$$
u \mid\left\{z_{q+2}=\cdots=z_{n}=0\right\} \text {. Q.E.D. }
$$

(1.7) Proof OF Theorem I FOR $k=1$. Let $D$ be the open subset of $C^{n}$ where $u$ is defined. Fix $x_{0} \in D$ and $c \geqq 0$. We are going to prove the following by induction on $q$.

If $V$ is an analytic subset of dimension $\leqq q$ in an open neighborhood $U$ of $x_{0}$ in $D$, then there exists an open neighborhood $U^{\prime}$ of $x_{0}$ in $U$ such that $E_{c} \cap V \cap U^{\prime}$ is an analytic subset of $V \cap U^{\prime}$.

It is clear that $(\dagger)_{0}$ is true. Assume $(\dagger)_{q-1}$. By shrinking $U$ and by replacing $V$ by a larger analytic set of dimension $\leqq q$, we can assume without loss of generality that there exists a proper holomorphic map $f: U \rightarrow D^{\prime} \times B$ (where $D^{\prime}$ is a Stein domain in $C^{q}$ and $B$ is the open unit ball in $C^{n-q}$ ) such that $V=f^{-1}\left(D^{\prime} \times\{0\}\right)$ and $f$ is regular on a dense subset of $V$. Let $u^{\prime}=f_{*} u$. Let $V=\bigcup_{i=1}^{p} V_{i}$ be the decomposition of $V$ into branches. Let $c_{i}=\inf \left\{n(u, x) \mid x \in V_{i}\right\}$. We can assume without loss of generality that $c \leqq c_{i}$ for $1 \leqq i \leqq l$ and $c>c_{i}$ for $l<i \leqq p$. We can assume also that $l<p$, otherwise $E_{c} \cap V=V$ is an analytic set. Let $\lambda_{i}$ be the number of sheets of $V_{i}$ over $D^{\prime} \times\{0\}$ and let

$$
\begin{aligned}
e & =\operatorname{Min}\left\{c-c_{i} \mid l<i \leqq p\right\}, \\
d & =e+\sum_{i=1}^{p} \lambda_{i} c_{i} \\
E_{d}^{\prime} & =\left\{x \in D^{\prime} \times B \mid n\left(u^{\prime}, x\right) \geqq d\right\} .
\end{aligned}
$$


By (1.6), $E_{d}^{\prime} \cap\left(D^{\prime} \times\{0\}\right)$ is contained in an analytic set $W$ of dimension $<q$ in $D^{\prime} \times\{0\}$. Let $S$ be the set of points of $V$ where $f$ is not regular. Let $T=f^{-1} f(S) \cup f^{-1}(W)$. Since

$$
\operatorname{dim} T<q \text { and } E_{c} \cap V=\left(\bigcup_{i=1}^{l} V_{i}\right) \cup\left(E_{c} \cap T\right),
$$

we conclude that $(\dagger)_{q}$ holds. Q.E.D.

2. We are going to sketch the proof of Theorem II for the case $X=\Delta^{2}, A=\Delta \times\{0\}, G=\Delta_{1 / 2} \times \Delta$, where $\Delta$ is the open unit 1-disc.

The following theorem is proved by using Hörmander's $L^{2}$ estimates for $\bar{\partial}$ in more or less the same way as the proof given in $[10]$.

(2.1) THEOREM. Let $Y$ be a complex manifold, $B$ be an analytic subset of codimension $\geqq 1$ in $Y$, and $H$ be an open subset of $Y$ which intersects every branch of $B$ of codimension 1. Then every plurisubharmonic function on $(Y-B) \cup H$ can be extended to a plurisubharmonic function on $Y$.

Actually the following more general theorem is true.

(2.1)' THEOREM. Let $Y$ be a complex manifold, $B$ be an analytic subset of codimension $\geqq k$ in $Y$, and $H$ be an open subset of $Y$ whose intersection with every branch of $B$ of codimension $k$ is nonempty and irreducible. Then every closed positive $(k, k)$ current on $(Y-B) \cup H$ can be extended to a closed positive $(k, k)$ current on $Y$.

The proof of (2.1)' makes use of [10] and (2.1) and projections, but we do not need (2.1)'.

Let $\omega$ be the Kähler form of $M$. By [10] and (2.1),

$$
f^{*} \omega+(\sqrt{-1} / 2) d z_{1} \wedge d \bar{z}_{1}+(\sqrt{-1} / 2) d z_{2} \wedge d \bar{z}_{2}
$$

can be extended to a closed positive $(1,1)$ current $u$ on $\Delta^{2}$. Let $V$ be the graph of $f$ and let $\bar{V}$ be the topological closure of $V$ in $\Delta^{2} \times M$.

(2.2) Lemma. If $x_{0} \in \Delta^{2}$ and $n\left(u, x_{0}\right)=0$, then $h^{2}\left(\left(\left\{x_{0}\right\} \times M\right) \cap \bar{\eta}\right)=0$, where $h^{2}$ denotes the Hausdorff 2-measure.

Proof. It suffices to show that every point of $M$ admits an open neighborhood $U$ such that $h^{2}\left(\left(\left\{x_{0}\right\} \times U\right) \cap \bar{V}\right)=0$. We can assume that $U$ is an open subset of $\boldsymbol{C}^{n}$ and $\omega \mid U$ is so close to the Euclidean Kähler form that, by Bishop's result [1, p. 299, Corollary 1 to Theorem 2],

$$
\begin{aligned}
& \text { if } B^{n+2}(\tilde{x}, r) \subset \Delta^{2} \times U \text { and } \Gamma \text { is a complex curve in } \\
& B^{n+2}(\tilde{x}, r)-\Delta \times\{0\} \times U \text { with } \tilde{x} \in \Gamma \text {, then } \mu(\Gamma) \geqq c r^{2},
\end{aligned}
$$

where $B^{n+2}(\tilde{x}, r)$ is the open ball of complex dimension $n+2$ with center 
$\tilde{x}$ and radius $r, \mu(\Gamma)$ is the volume of $\Gamma$ with respect to the Kähler metric of $M$ and the Euclidean metric of $\Delta^{2}$, and $c$ is a positive constant.

Fix $r_{0}>0$ with $B^{2}\left(x_{0}, r_{0}\right) \subset \subset \Delta^{2}$ and fix a relatively compact open neighborhood $W$ of $[0,1]$ in $\boldsymbol{P}_{1}-\{[1,0]\}$. For $x \in \Delta^{2}$ close to $x_{0}$, define the following measure $m_{x}$ on $\left(\Delta^{2} \times U\right) \cap V$ :

$$
\left.m_{x}(E)=\int_{T \in W} \mu\left(\left((x+T) \cap B^{2}\left(x, r_{0}\right)\right) \times U\right) \cap E\right) d T,
$$

where $T=\left[z_{1}, z_{2}\right] \in W$ is identified with the complex line passing through 0 and $\left(z_{1}, z_{2}\right)$. By using the regularization of plurisubharmonic functions and Fatou's lemma as in [5], we conclude that

$$
m_{x_{0}}\left(\left(\Delta^{2} \times U\right) \cap V\right)<\infty \text {. }
$$

We claim that

$$
\begin{aligned}
& \text { If } \tilde{x} \in\left(\left\{x_{0}\right\} \times U\right) \cap \bar{V} \text { and } B^{n+2}(\tilde{x}, r) \subset \Delta^{2} \times U \text { with } \\
& r<r_{0} \text {, then } m_{x_{0}}\left(B^{n+2}(\tilde{x}, r) \cap V\right) \geqq c r^{2} .
\end{aligned}
$$

There exists $\tilde{x}_{j}=\left(x_{j}, y_{j}\right) \in\left(\Delta^{2} \times U\right) \cap V$ approaching $\tilde{x}$ such that $f$ is holomorphic at $x_{j}$. By $\left({ }^{*}\right), m_{x_{j}}\left(B^{n+2}\left(\tilde{x}_{j}, r\right) \cap V\right) \geqq c r^{2}$. Since $n\left(u, x_{0}\right)=0$, for any given $\varepsilon>0$, when $j$ is sufficiently large and $\delta$ is sufficiently small,

$$
m_{x_{j}}\left(\left(B^{2}\left(x_{j}, \delta\right) \times U\right) \cap V\right)<\varepsilon .
$$

Now (\#) follows from the theorem of bounded convergence, and $h^{2}\left(\left(\left\{x_{0}\right\} \times U\right) \cap \bar{V}\right)=0$ follows from $(\dagger \dagger)$ and (\#). Q.E.D.

(2.3) Proof of Theorem II FOR THE SPECIAL CASE. By the regularization of plurisubharmonic functions and Fatou's lemma together with Bishop's theorem [1], as in [5] we conclude that

(\#\#) for every $z_{1} \in \Delta, f \mid\left\{z_{1}\right\} \times(\Delta-\{0\})$ can be extended
to a holomorphic map from $\left\{z_{1}\right\} \times \Delta$ to $M$.

By (2.2), for $\tilde{x}=(x, y) \in(\Delta \times\{0\}) \times M$ with $n(u, x)=0$, we can find an open polydisc neighborhood $\Delta^{\prime} \times \Delta^{\prime \prime}$ of $x$ in $\Delta^{2}$, an open neighborhood $U$ of $y$ in $M$, and a holomorphic function $w$ on $\Delta^{\prime} \times \Delta^{\prime \prime} \times U$ such that the map $g$ from $\left(\Delta^{\prime} \times \Delta^{\prime \prime} \times U\right) \cap \bar{V}$ to $\Delta^{\prime} \times \Delta^{\prime \prime} \times \Delta$ defined by $\left(z_{1}, z_{2}, w\right)$ is proper. Let

$$
Z=g\left(g^{-1}\left(\Delta^{\prime} \times\left(\Delta^{\prime \prime}-\{0\}\right) \times \Delta\right)\right) .
$$

Then $Z$ is an analytic subset of pure codimension 2 in $\Delta^{\prime} \times\left(\Delta^{\prime \prime}-\{0\}\right) \times$ $\Delta$. By $(\# \#)$, for every $z_{1} \in \Delta^{\prime}, Z \cap\left(\left\{z_{1}\right\} \times\left(\Delta^{\prime \prime}-\{0\}\right) \times \Delta\right)$ can be extended to an analytic subset of $\left\{z_{1}\right\} \times \Delta^{\prime \prime} \times \Delta$. Hence $Z$ can be extended to an analytic subset of $\Delta^{\prime} \times \Delta^{\prime \prime} \times \Delta$. It follows that $\left(\Delta^{\prime} \times \Delta^{\prime \prime} \times U\right) \cap \bar{V}$ is an analytic set. 
By Theorem I, the set of all $x \in \Delta^{2}$ with $n(u, x)>0$ is at most countable. It follows that, for every compact subset $K$ of $\Delta^{2}$, we can find an open neighborhood $L$ of $K$ in $\Delta^{2}$ and an open neighborhood $Q$ of the boundary $\partial L$ of $L$ in $\Delta^{2}$ such that $\partial L$ is a compact $C^{\infty}$ submanifold of $\Delta^{2}$ and $f \mid Q-\Delta \times\{0\}$ can be extended to a holomorphic map from $Q$ to $M$. Since $u$ is $C^{\infty}$ on $Q$, by the regularization of plurisubharmonic functions and Fatou's lemma together with Bishop's theorem, as in [5] we conclude that $f \mid L-\Delta \times\{0\}$ can be extended to a meromorphic map from $L$ to M. Q.E.D.

\section{REFERENCES}

1. E. Bishop, Conditions for the analyticity of certain sets, Michigan Math. J. 11 (1964), 289-304. MR 29 \#6057.

2. E. Bombieri, Algebraic values of meromorphic maps, Invent. Math. 10 (1970), 267-287.

3. - Addendum to my paper: Algebraic values of meromorphic maps, Invent. Math. 11 (1970), 163-166.

4. H. Federer, Geometric measure theory, Die Grundlehren der math. Wissenschaften, Band 153, Springer-Verlag, New York, 1969. MR 41 \# 1976.

5. P. A. Griffiths, Two theorems on extensions of holomorphic mappings, Invent. Math. 14 (1971), 27-62. MR 45 \#2202.

6. F. R. Harvey and J. R. King, On the structure of positive currents, Invent. Math. 15 (1972), 47-52. MR 45 \#5409.

7. J. King, The currents defined by analytic varieties, Acta Math. 127 (1971), 185-220.

8. P. Lelong, Plurisubharmonic functions and positive differential forms, Gordon and Breach, New York, 1969. MR 39 \#4436.

9. B. Shiffman, Extension of positive line bundles and meromorphic maps, Invent. Math. 15 (1972), 332-347.

10. Y.-T. Siu, A Thullen type extension theorem for positive holomorphic vector bundles, Bull. Amer. Math. Soc. 78 (1972), 775-776.

11. H. Skoda, Sous-ensembles analytiques d'ordre fini ou infini dans $\boldsymbol{C}^{n}$, Bull. Soc. Math. France 100 (1972), 353-408.

12. P. Thie, The Lelong number of a point of a complex analytic set, Math. Ann. 172 (1967), 269-312. MR 35 \#5661.

Department of Mathematics, Yale University, New Haven, Connecticut 06520 